Journal of

Applied

Crystallography

ISSN 1600-5767

\title{
Crossing the boundary between face-centred cubic and hexagonal close packed: the structure of nanosized cobalt is unraveled by a model accounting for shape, size distribution and stacking faults, allowing simulation of XRD, XANES and EXAFS
}

\section{Alessandro Longo, Luisa Sciortino, Francesco Giannici and Antonino Martorana}

J. Appl. Cryst. (2014). 47, 1562-1568

Copyright (C) International Union of Crystallography

Author(s) of this paper may load this reprint on their own web site or institutional repository provided that this cover page is retained. Republication of this article or its storage in electronic databases other than as specified above is not permitted without prior permission in writing from the IUCr.

For further information see http://journals.iucr.org/services/authorrights.html

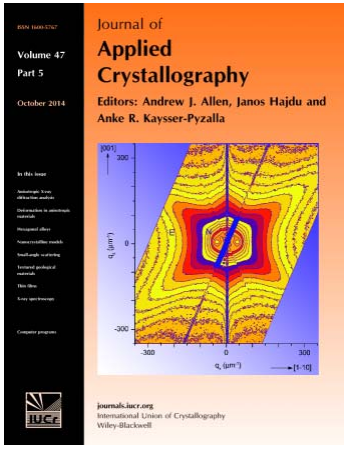

Many research topics in condensed matter research, materials science and the life sciences make use of crystallographic methods to study crystalline and non-crystalline matter with neutrons, X-rays and electrons. Articles published in the Journal of Applied Crystallography focus on these methods and their use in identifying structural and diffusioncontrolled phase transformations, structure-property relationships, structural changes of defects, interfaces and surfaces, etc. Developments of instrumentation and crystallographic apparatus, theory and interpretation, numerical analysis and other related subjects are also covered. The journal is the primary place where crystallographic computer program information is published.

Crystallography Journals Online is available from journals.iucr.org 
Journal of

Applied

Crystallography

ISSN 1600-5767

Received 10 June 2014

Accepted 8 July 2014

\section{Crossing the boundary between face-centred cubic and hexagonal close packed: the structure of nanosized cobalt is unraveled by a model accounting for shape, size distribution and stacking faults, allowing simulation of XRD, XANES and EXAFS}

\author{
Alessandro Longo, ${ }^{\text {a,b }}$ Luisa Sciortino, ${ }^{\mathrm{c}}$ Francesco Giannici $^{\mathrm{c}}$ and Antonino \\ Martorana $^{\mathrm{C} *}$ \\ aIstituto per lo Studio dei Materiali Nanostrutturati, CNR, Via Ugo La Malfa 153, Palermo, I-90146, \\ Italy, ${ }^{\mathbf{b}}$ Netherlands Organization for Scientific Research (NWO), 6 rue Jules Horowitz, Grenoble, \\ BP220, 38043, France, and ${ }^{\mathbf{C}}$ Dipartimento di Fisica e Chimica, Università di Palermo, Parco \\ D'Orleans II, Palermo, I-90128, Italy. Correspondence e-mail: antonino.martorana@unipa.it
}

\begin{abstract}
The properties of nanostructured cobalt in the fields of magnetic, catalytic and biomaterials depend critically on Co close packing. This paper reports a structural analysis of nanosized cobalt based on the whole X-ray diffraction (XRD) pattern simulation allowed by the Debye equation. The underlying structural model involves statistical sequences of cobalt layers and produces simulated XRD powder patterns bearing the concurrent signatures of hexagonal and cubic close packing (h.c.p. and f.c.c.). Shape, size distribution and distance distribution between pairs of atoms are also modelled. The simulation algorithm allows straightforward fitting to experimental data and hence the quantitative assessment of the model parameters. Analysis of two samples having, respectively, h.c.p. and f.c.c. appearance is reported. Extended X-ray absorption fine-structure (EXAFS) and X-ray absorption near-edge structure (XANES) spectra are simulated on the basis of the model, giving a tool for the interpretation of structural data complementary to XRD. The outlined structural analysis provides a rigorous structural basis for correlations with magnetic and catalytic properties and an experimental reference for ab initio modelling of these properties.

\section{Introduction}

The boundary between hexagonal close-packed (h.c.p.) and face-centred cubic (f.c.c.) polytypes for most nanostructured metals displaying a close-packing (CP) arrangement is a no man's land where, depending on particle size, preparation route or processing protocol, h.c.p. and cubic close-packed (c.c.p.) sequences of basal atomic layers (labelled $A, B, C$, according to the usual $\mathrm{CP}$ arrangement notation) can be present at the same time. In this respect, the case of cobalt deserves much interest, concerning the outcome of different synthetic protocols (Puntes et al., 2001; McHenry et al., 1994; Leslie-Pelecky et al., 1998) and the modification of the CP arrangement accomplished by various techniques such as ball milling (Sort et al., 2004), thermal treatment (Speight et al., 2009) or irradiation with heavy atoms (Sprouster et al., 2009). The polytypism of cobalt has also been the subject of theoretical analysis, arguing that the f.c.c. and h.c.p. structures are originated from a parent polytypic structure where ordered domains grow at the expense of alternating disordered regions (Tolédano et al., 2001).

A detailed structural analysis of nanosized cobalt is particularly relevant, taking into account that a great many applications in the fields of magnetic (Chakroune et al., 2003; Margeat et al., 2005; Lu et al., 2007; Qiao et al., 2007; Zhao et al., 2010), catalytic (de la Peña O'Shea et al., 2009; Li et al., 2011; Gnanamani et al., 2013) and biomaterials (Jun et al., 2008; Xu et al., 2008; Pankhurst et al., 2009) depend critically on Co close packing. In particular, it is well established that the h.c.p. arrangement gives rise to higher magnetic anisotropy in magnetic recording cobalt materials (Sort et al., 2003; Sokalski et al., 2011; Schio et al., 2013); h.c.p. cobalt shows better performance also as a component in Fischer-Tropsch catalysts, in a few cases stimulating interesting structural studies based on X-ray diffraction (XRD) pattern simulation (Ducreux et al., 2009) and ab initio computational modelling (Liu et al., 2013). Reference to a detailed structural analysis 
can also be important in view of the complementarity of XRD with local probes like X-ray absorption spectroscopy (Sprouster et al., 2009; Sciortino et al., 2011) and ${ }^{59}$ Co solid state NMR (Speight et al., 2009), and for ab initio modelling of magnetic properties of cobalt and cobalt nanoalloys (Ferrando et al., 2008; Barcaro et al., 2011; Hakamada et al., 2012).

In most of the cited literature the reported XRD patterns of nanosized cobalt present the concurrent signatures of h.c.p. and c.c.p. sequences, which are routinely attributed to the presence, in different ratios, of both the h.c.p. and f.c.c. polytypes. In some cases the importance of a deeper insight into the structure of nanosized Co was recognized, leading to the simulation of XRD patterns on the basis of a suitable structural model (Ducreux et al., 2009; Sokalski et al., 2011). In particular, Sokalski et al. (2011) assumed that the position of a Co basal layer is statistically determined by the three preceding layers and developed, according to Jagodzinski's formalism (Jagodzinski, 1949), a model with Reichweite $s=3$.

In this paper we report a structural model of nanosized cobalt allowing for (i) a size distribution of spherical crystallites, (ii) CP sequences where four-layer blocks influence in a probabilistic way the occurrence of the next layer and (iii) a distribution of distances between pairs of atomic centres. The XRD simulation is carried out in the framework of the Debye equation (Debye, 1915), which in the past was adopted only sporadically (Cervellino et al., 2003; Niederdraenk et al., 2007; Longo \& Martorana, 2008; Ducreux et al., 2009; Rempel \& Magerl, 2010), probably because of the cumbersome requirement of computing resources. In this respect, the Debye equation approach is particularly demanding when dealing with structural disorder, which in some cases has been tackled by averaging over all possible disordered structures (Niederdraenk et al., 2007; Ducreux et al., 2009; Rempel \& Magerl, 2010). An alternative to this latter procedure is represented by the 'parent stack' approach (Longo \& Martorana, 2008), which allows a viable use of standard fitting algorithms and thereby a straightforward estimate of the model parameters. As shown in this paper, the optimized parameters can be used to simulate extended X-ray absorption fine-structure (EXAFS) and X-ray absorption near-edge structure (XANES) spectra and perform a comparison with experimental data.

\section{Experimental}

\subsection{Synthetic procedures}

The syntheses were carried out by thermal decomposition of $\mathrm{Co}_{2}(\mathrm{CO})_{8}(\geq 95 \%$, Fluka), according to a procedure leading to the formation of free cobalt particles coated by capping molecules. The synthesis route involved three steps: (i) $1.4 \times$ $10^{-3} \mathrm{~mol}(0.5 \mathrm{~g})$ of $\mathrm{Co}_{2}(\mathrm{CO})_{8}$ and the capping agent octadecylamine (ODA) were put into $25 \mathrm{ml}$ of organic solvent at boiling temperature under continuous argon flow; (ii) the solution was kept for $3 \mathrm{~h}$ under reflux; (iii) the samples were dried under vacuum, so obtaining the powder samples. Two setups were exploited, giving rise to different Co powder samples: $\mathrm{Co} / \mathrm{ODA}=500: 1$ [molar ratio $(\mathrm{Co}$ atoms $) /(\mathrm{ODA}$ molecules)] in heptane (boiling point $371 \mathrm{~K}$ ), for sample $\mathrm{H}$; $\mathrm{Co} / \mathrm{ODA}=200: 1$ in mesytilene (boiling point $438 \mathrm{~K}$ ), for sample C.

\subsection{High-resolution X-ray diffraction}

High-resolution X-ray diffraction patterns were recorded at the ID31 beamline of the European Synchrotron Radiation Facility (ESRF) in transmission geometry at a constant scanning speed with incident wavelength $\lambda=0.40000 \AA$.

\subsection{X-ray absorption}

X-ray absorption spectroscopy was performed in transmission geometry at beamline BM26A of the ESRF using an Si(111) double-crystal monochromator. A cobalt foil was placed downstream of the sample for energy calibration. The spectra were acquired at $80 \mathrm{~K}$ using a liquid nitrogen cryostat.

\section{Structural model and XRD simulation}

The model powder pattern is calculated by taking the average of a statistical ensemble of faulted crystallites that is generated from a fictitious structure called the 'parent stack' (Longo \& Martorana, 2008). The latter is constituted by atoms placed in

$$
\mathbf{r}_{\xi \eta \zeta}=\xi \mathbf{a}+\eta \mathbf{b}+\zeta \mathbf{c},
$$

where $\xi, \eta, \zeta$ are integers and $\mathbf{a}, \mathbf{b}, \mathbf{c}$ are hexagonal crystallographic axes $\left(|\mathbf{a}|=|\mathbf{b}| \neq|\mathbf{c}|, \alpha=\beta=90^{\circ}, \gamma=120^{\circ}\right)$.

The shape function of the parent stack is defined as

$$
\varphi(\mathbf{r})= \begin{cases}1 & \text { inside the parent stack } \\ 0 & \text { outside }\end{cases}
$$

The interatomic vectors of the parent stack are given by

$$
\mathbf{t}_{k l m}=k \mathbf{a}+l \mathbf{b}+m \mathbf{c}
$$

where $k, l, m$ are integers; the distance multiplicity $\mu\left(\mathbf{t}_{k l m}\right)$ is given by the number of times a given interatomic vector occurs:

$$
\mu\left(\mathbf{t}_{k l m}\right)=(\mathbf{a} \cdot \mathbf{b} \wedge \mathbf{c})^{-1} \int_{V_{\infty}} \mathrm{d} \mathbf{r} \varphi(\mathbf{r}) \varphi\left(\mathbf{r}+\mathbf{t}_{k l m}\right) .
$$

Then, equation (4) represents the volume common to the shape function and its ghost translated by $\mathbf{t}_{k l m}$, divided by the volume per atom.

Each element of the statistical ensemble representing the myriad of crystallites with different stacking sequences of basal layers is obtained from the parent stack by the slip vectors

$$
\mathbf{r}_{A}=-\frac{1}{3} \mathbf{a}-\frac{1}{3} \mathbf{b}, \quad \mathbf{r}_{B}=\frac{1}{3} \mathbf{a}, \quad \mathbf{r}_{C}=\frac{1}{3} \mathbf{b},
$$

where the subscripts $A, B, C$ denote the three allowed compact packing positions. The vectors (5) bring about the shift of the whole layer of atoms at height $\zeta$ [equation (1)] and are the shortest vectors whose sequence along complies with the $\mathrm{CP}$ requirement; periodic sequences produce regular closepacked structures (say, $\mathbf{r}_{A} \mathbf{r}_{B} \mathbf{r}_{A} \mathbf{r}_{B} \ldots$ for h.c.p. and $\mathbf{r}_{A} \mathbf{r}_{B} \mathbf{r}_{C}$ $\mathbf{r}_{A} \mathbf{r}_{B} \mathbf{r}_{C} \ldots$ for f.c.c.); also, random sequences are possible, with 
the constraint that consecutive equal shifts (say, $\mathbf{r}_{A} \mathbf{r}_{A}$ ) are forbidden.

The faulted sequences can be completely uncorrelated, or a range of correlation $s$ can hold, defining how many foregoing layers determine the statistical occurrence of the next one. The latter case is treated with Kakinoki's matrix formalism (Kakinoki, 1967), allowing for probabilistic events consisting of $s$-layer building blocks. According to the CP constraint, there are $\tau=3\left(2^{s-1}\right)$ distinct blocks: once a given $s$ range has been assumed, the probability that the $i$ th block $(i=1 \ldots \tau)$ continues with a layer in the $A, B$ or $C$ position is defined and thereby the frequency of occurrence of the $i$ th block can be calculated. To illustrate, in the case $s=3$, there are 12 different $s$-layer blocks:

$$
\begin{array}{lll}
B C A \equiv 1(\mathrm{c}) & C A B \equiv 5(\mathrm{c}) & A B C \equiv 9(\mathrm{c}) \\
A C A \equiv 2(\mathrm{~h}) & B A B \equiv 6(\mathrm{~h}) & C B C \equiv 10(\mathrm{~h}) \\
A B A \equiv 3(\mathrm{~h}) & B C B \equiv 7(\mathrm{~h}) & C A C \equiv 11(\mathrm{~h}) \\
C B A \equiv 4(\mathrm{c}) & A C B \equiv 8(\mathrm{c}) & B A C \equiv 12(\mathrm{c})
\end{array}
$$

These are labelled as $\mathrm{h}$ (hexagonal) or c (cubic), according to the type of close packing. The elements $p_{i j}$ of the $\tau \times \tau$ matrix $\mathbf{P}$ give the probability of $i-j$ block concatenation: for example, the first block $B \underline{C A}$ has a probability $p_{15}$ of being concatenated to the fifth block $\underline{C A} B$. Only blocks ending and starting with the same underscored sequence are concatenated (so, $B C A$ and $A B C$ are not, and the corresponding probability $p_{19}$ is null). This is equivalent to the statement that $p_{15}$ represents the probability that the $B C A$ block continues with $B$, according to the sequence $B C A \_B$ or, making reference to close-packing labels, to a c_c sequence. Taking into account symmetry-equivalent sequences, the $\mathbf{P}$ matrix has a block structure,

$$
\mathbf{P}=\left(\begin{array}{lll}
\mathbf{P}^{0} & \mathbf{P}^{1} & \mathbf{P}^{2} \\
\mathbf{P}^{2} & \mathbf{P}^{0} & \mathbf{P}^{1} \\
\mathbf{P}^{1} & \mathbf{P}^{2} & \mathbf{P}^{0}
\end{array}\right)
$$

where the elements of $\mathbf{P}^{0}$ are all zero and

$$
\mathbf{P}^{1}=\left(\begin{array}{cccc}
\alpha & 0 & 0 & 0 \\
\beta & 0 & 0 & 0 \\
0 & 1-\beta & 0 & 0 \\
0 & 1-\alpha & 0 & 0
\end{array}\right), \quad \mathbf{P}^{2}=\left(\begin{array}{cccc}
0 & 0 & 1-\alpha & 0 \\
0 & 0 & 1-\beta & 0 \\
0 & 0 & 0 & \beta \\
0 & 0 & 0 & \alpha
\end{array}\right)
$$

Then, $\alpha$ defines a c_c probability, $\beta$ is relative to $\mathrm{h} \_\mathrm{c},(1-\alpha)$ accounts for c_h and $(1-\beta)$ for $\mathrm{h} \_$h. The $m$ th power of $\mathbf{P}$, $(\mathbf{P})^{m}$, represents the probabilities relative to the $m$ th neighbours and $(\mathbf{P})^{0}=\mathbf{1}$ (the identity $\tau \times \tau$ matrix) completes the definition of the powers of $\mathbf{P}$.

The frequency of occurrence of the $i$ th block is given by

$$
f_{i}=\sum_{j} f_{j} p_{j i}
$$

Solving the linear and homogeneous system (9) yields the values of $f_{i}(i=1,2, \ldots, 12)$, which assume only two distinct values, one for the $\mathrm{h}$ blocks and one for the c blocks of equation (6). The overall frequency values relative to, respectively, the $\mathrm{h}$ and $\mathrm{c}$ blocks are then

$$
f_{\mathrm{h}}=\frac{1-\alpha}{1-\alpha-\beta}, \quad f_{\mathrm{c}}=\frac{\beta}{1-\alpha-\beta} .
$$

The interatomic vector between atoms belonging to the last layers of the $i$ th and $j$ th blocks, $m$ layers apart from each other, is given by

$$
\mathbf{t}_{k l m}^{i j}=\mathbf{t}_{k l m}+\left(\mathbf{r}_{j}-\mathbf{r}_{i}\right)
$$

where $\mathbf{t}_{k l m}$ is defined by equation (3) and $\mathbf{r}_{j}, \mathbf{r}_{i}$ are two of the shift vectors defined in equation (5).

According to the Debye (1915) equation, the diffraction intensity for ordered crystallites is given by

$$
I(q)=\sum_{\mathbf{t}} \mu(\mathbf{t}) \frac{\sin 2 \pi q|\mathbf{t}|}{2 \pi q|\mathbf{t}|},
$$

where $q$ is the length of the scattering vector $(q=2 \sin \theta / \lambda, \theta$ being half the scattering angle). The sum is extended to all the distinct interatomic vectors, and $\mu(\mathbf{t})$, defined in equation (4), accounts for the number of times a given distance vector $\mathbf{t}$ is present in the structure. The corresponding equation relative to a faulted structure involves the average over all the probabilistic configurations:

$$
I_{k l m}=\sum_{i, j} f_{i} p_{i j}^{m} \frac{\sin 2 \pi q\left|\mathbf{t}_{k l m}^{i j}\right|}{2 \pi q\left|\mathbf{t}_{k l m}^{i j}\right|},
$$

where $p_{i j}^{m}$ represents the $(i j)$ th element of the $(\mathbf{P})^{m}$ matrix. Taking into account that the actual particles are generated by rigid translation of whole layers of the parent stack, the overall intensity is then obtained by summing over all the $\mathbf{t}_{\mathrm{klm}}$ distances of the parent stack, weighted by the distance multiplicity given by equation (4).

In nanostructured materials, microstrains produce a finitewidth distribution of distances, involving a broadening of the powder diffraction peaks that increases as a function of $q$ (Longo \& Martorana, 2008). The model assumes a spherically symmetric Gaussian distribution whose width is a linear function of the distance between pairs of scatterers:

$$
\Phi_{\mathbf{t}_{k l m}}=\sigma_{\mathbf{t}_{k l m}}^{-3}(2 \pi)^{-3 / 2} \exp \left[-\left(\mathbf{t}-\mathbf{t}_{k l m}\right)^{2} / 2 \sigma_{\mathbf{t}_{k l m}}^{2}\right],
$$

where

$$
\sigma_{\mathbf{t}_{k l m}}^{2}=\left(\left|\mathbf{t}_{k l m}\right| /\left|\mathbf{t}_{0}\right|\right) \sigma_{\mathbf{t}_{0}}^{2}
$$

$\left|\mathbf{t}_{0}\right|$ is a reference unit length and $\sigma_{\mathbf{t}_{0}}$ is the corresponding width of the distance distribution. As a consequence of the assumed distance distribution, each term of the Debye equation is multiplied by the Fourier transform of equation (14):

$$
\varphi_{\mathbf{t}_{k l m}}(q)=\exp \left(-2 \pi^{2} q^{2} \sigma_{\mathbf{t}_{k l m}}^{2}\right)
$$

Finally, a lognormal size distribution was assumed (Popa \& Balzar, 2002). For spherical crystallites, the distribution of radii is then

$$
\Psi(R)=\frac{1}{R[2 \pi \ln (1+c)]^{1 / 2}} \exp \left\{-\frac{\ln \left[\left(R / R_{\mathrm{av}}\right)(1+c)^{1 / 2}\right]^{2}}{2 \ln (1+c)}\right\} .
$$


In equation (17) $R_{\mathrm{av}}$ is the average radius and $c=\left(\sigma_{R} / R_{\mathrm{av}}\right)^{2}$, where $\sigma_{R}$ is the distribution standard deviation.

\section{Results and discussion}

\subsection{XRD analysis}

Fig. 1 reports a grid of simulated patterns corresponding to the different $(\alpha, \beta)$ values relative to the model $s=3$. From inspection, it is evident that the model is able to reproduce the simultaneous presence of the features that are usually attributed to separate h.c.p. and f.c.c. phases in the XRD patterns reported in the literature.

The properties of nanosized Co depend crucially not only on the compact packing sequences but also on the shape of crystallites (Diehl et al., 2001; Xu et al., 2007; Liu et al., 2008; Srivastava et al., 2010). To show how the pattern can be modified as a function of shape, Fig. 2 reports the simulated patterns corresponding to the $(\alpha, \beta)$ pairs of the third row of Fig. 1, relative to rods, spheres and discs with the same volume; it is evident that the crystallite shape strongly affects the features of the diffraction pattern, as previously demonstrated for ordered cobalt nanoparticles (Puntes et al., 2002).

In our approach the XRD Debye simulation can be easily linked to an optimization algorithm (we used the CERN package MINUIT; http:/seal.web.cern.ch/seal/work-packages/ mathlibs/minuit/home.html), to get the best fitting of a model of structural disorder to experimental data. Here we report the structural analysis of the two synthesized $\mathrm{H}$ and $\mathrm{C}$ cobalt samples. The model for these samples allows for a correlation range $s=4$, henceforth depending on the four probability parameters, $\alpha, \beta, \gamma, \delta$, described in Table 1 . The case $s=3$,

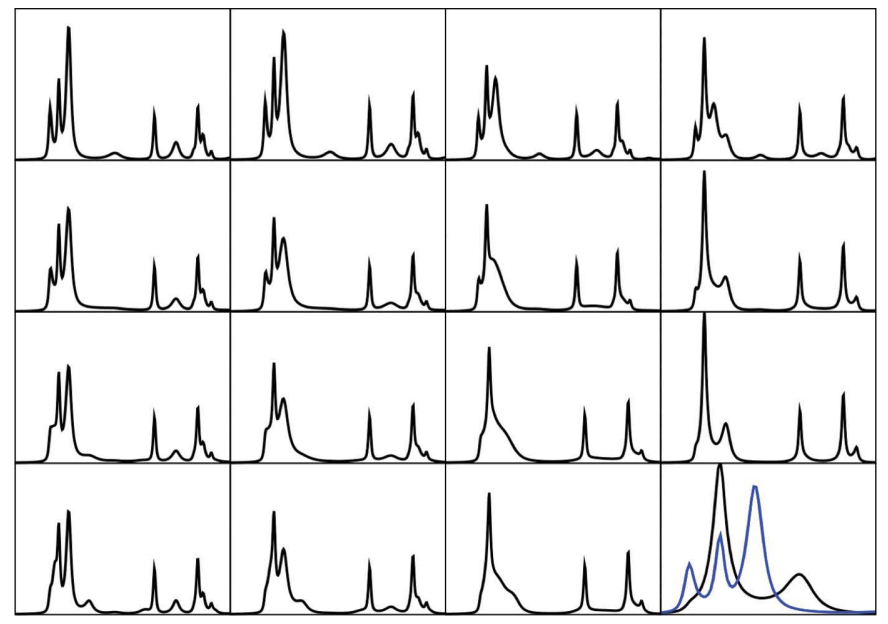

Figure 1

Simulated powder patterns of Co, model $s=3$. Starting from $(\alpha, \beta)=(0.2$, 0.8 ) in the left lower corner, $\alpha$ increases rightwards and $\beta$ decreases upwards with a step of 0.2 . In the top right corner, the signatures of both close-packing arrangements are particularly evident. The pattern in the top left corner has the most h.c.p. character, while the maximum f.c.c. character is exhibited at the bottom right. To help discriminate the h.c.p. from the c.c.p. signatures, the top left pattern is plotted in blue over the latter in the $2 \theta$ range $10-14.5^{\circ}, \lambda=0.4 \AA$. All the other plots are in the $8-$ $24^{\circ} 2 \theta$ interval.
Table 1

Parameters ruling the statistical sequences for the model $s=4$.

For each parameter the respective $\mathrm{CP}$ sequence and an illustrative sample case is given.

\begin{tabular}{lll}
\hline CP probability & CP sequence & Example \\
\hline$\alpha$ & cc_c & $A B C A \_B$ \\
$\beta$ & hc_c & $A B A C \_B$ \\
$\gamma$ & hh_c & $A B A B \_C$ \\
$\delta$ & ch_c & $A B C B_{-} A$ \\
\hline
\end{tabular}

previously described, is recovered for $\alpha=\beta$ and $\gamma=\delta$, that is, when the cc_c, and hc_c sequences (and, respectively, hh_c and ch_c) are independent of the first basal layer $(A$, in the examples reported in Table 1).

Figs. 3 and 4 show the fittings relative to the XRD data of samples $\mathrm{H}$ and $\mathrm{C}$, respectively. From inspection, it is clear that in the $\mathrm{H}$ sample the signatures of h.c.p. packing are more evident, while in the $\mathrm{C}$ sample the c.c.p. sequences prevail. The fitting runs showed the necessity of an oxide component, whose presence and relative amount is confirmed by X-ray absorption spectra. The oxide component was simulated with Tchebischeff polynomials and pseudo-Voigt peak functions (turquoise line), giving intensity oscillations corresponding to amorphous or very small particles of $\mathrm{CoO}$ and $\mathrm{Co}_{3} \mathrm{O}_{4}$ (de la Peña O'Shea et al., 2009; Speight et al., 2009), probably constituting a thin shell enclosing the metal core (Lisiecki \& Pileni, 2011).

Table 2 reports the fitting results for $\mathrm{H}$ and $\mathrm{C}$. As can be observed, although at first sight one could get the impression of a large majority of cc blocks for sample $\mathrm{C}$ and of hh blocks for sample $\mathrm{H}$, the $f_{\mathrm{cc}}$ frequency is nearly equal to the $\operatorname{sum} f_{\mathrm{hh}}+$ $f_{\mathrm{hc}}+f_{\mathrm{ch}}$; similarly, $f_{\mathrm{hh}}$ is even less than the sum of $f_{\mathrm{cc}}+f_{\mathrm{hc}}+f_{\mathrm{ch}}$ for sample $\mathrm{H}$.

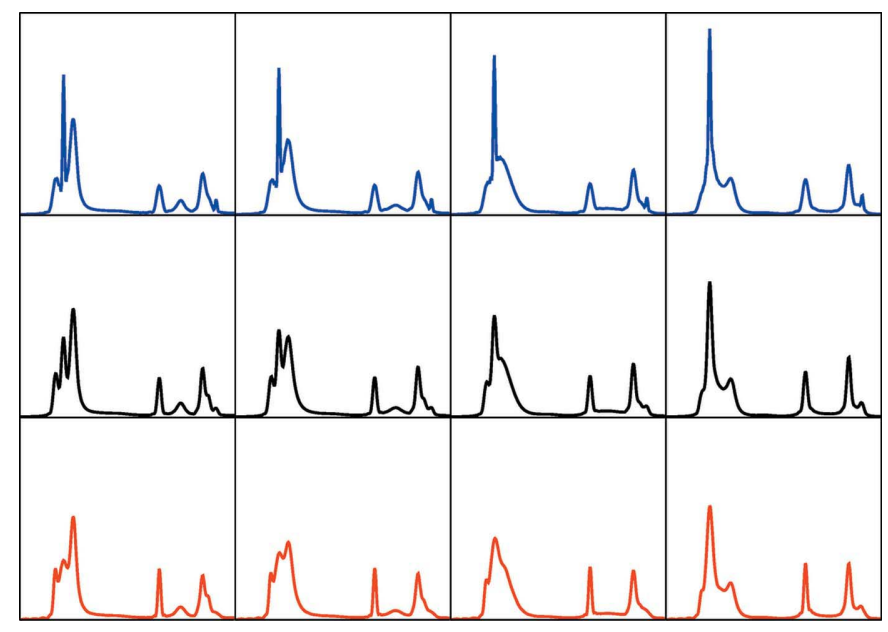

Figure 2

Powder patterns of rods (blue), spheres (black) and discs (red). The columns, from left to right, are relative to the $(\alpha, \beta)$ pairs $(0.2,0.4),(0.4$, $0.4),(0.6,0.4)$ and $(0.8,0.4)$, respectively. Rod diameter $d=50 \AA$ and height $h=125 \AA$, aspect ratio $h / d=2.5$. Spheres and discs have the same volume as rods; the discs' aspect ratio is $h / d=1 / 2.5$. $2 \theta$ range $\left(8-24^{\circ}\right), \lambda=$ $0.4 \AA$ Å. 
Table 2

Fitting results for samples $\mathrm{H}$ and $\mathrm{C}$.

Goodness of fit: $R_{\mathrm{H}}=0.064, R_{\mathrm{C}}=0.057$.

\begin{tabular}{llllllllllllll}
\hline & $a(\AA)$ & $c(\AA)$ & $R_{\mathrm{av}}(\AA) \dagger$ & $\sigma(\AA) \dagger$ & $\sigma_{\mathbf{t}_{0}}^{2}\left(\AA^{2}\right) \ddagger$ & $f_{\mathrm{cc}} \S$ & $\alpha \uparrow$ & $f_{\mathrm{hc}} \S$ & $\beta \uparrow$ & $f_{\mathrm{hh}} \S$ & $\gamma \uparrow$ & $f_{\mathrm{ch}} \S$ & $\delta \uparrow$ \\
\hline $\mathrm{H}$ & $2.5081(3)$ & $2.0407(2)$ & $30.4(6)$ & $18.4(8)$ & $0.0011(2)$ & $0.22(1)$ & $0.72(1)$ & $0.18(1)$ & $0.32(1)$ & $0.42(2)$ & $0.34(1)$ & $0.18(1)$ & $0.23(2)$ \\
$\mathrm{C}$ & $2.5080(2)$ & $2.0433(2)$ & $25.0(4)$ & $28.3(5)$ & $0.0015(2)$ & $0.51(1)$ & $0.81(2)$ & $0.17(2)$ & $0.57(1)$ & $0.15(2)$ & $0.58(1)$ & $0.17(2)$ & $0.48(2)$ \\
\hline
\end{tabular}

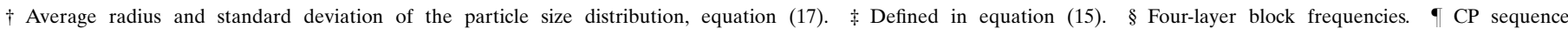
probabilities.

Fig. 5 reports the refined size distribution functions for the $\mathrm{H}$ and $\mathrm{C}$ samples; the larger average radius of the $\mathrm{H}$ sample and the shape of the respective radii distributions, showing a lesser fraction of very small crystallites, endorse the widely accepted conclusion that c.c.p. is favoured by a smaller particle size, even if for the investigated samples the preparation temperature and the interaction with the oxide phase could also have a role.

In principle, it is feasible to evaluate models with $s>4$, probably improving the fitting at the cost of correlations between an increasing number of probability parameters. Fitting runs with $s=3$ gave a significantly worse agreement [with reference to the normalized $\chi^{2}$, defined as $R=\chi^{2} /(n-$ $p$ ), where $n$ is the number of points and $p$ the number of parameters, we have $R_{s=3}=6.78, R_{s=4}=4.73$ for the H sample; $R_{s=3}=15.36, R_{s=4}=11.65$ for the C sample]. Transmission electron microscopy data did not evidence definitely anisometric particles, so that only fittings with spheres are reported.
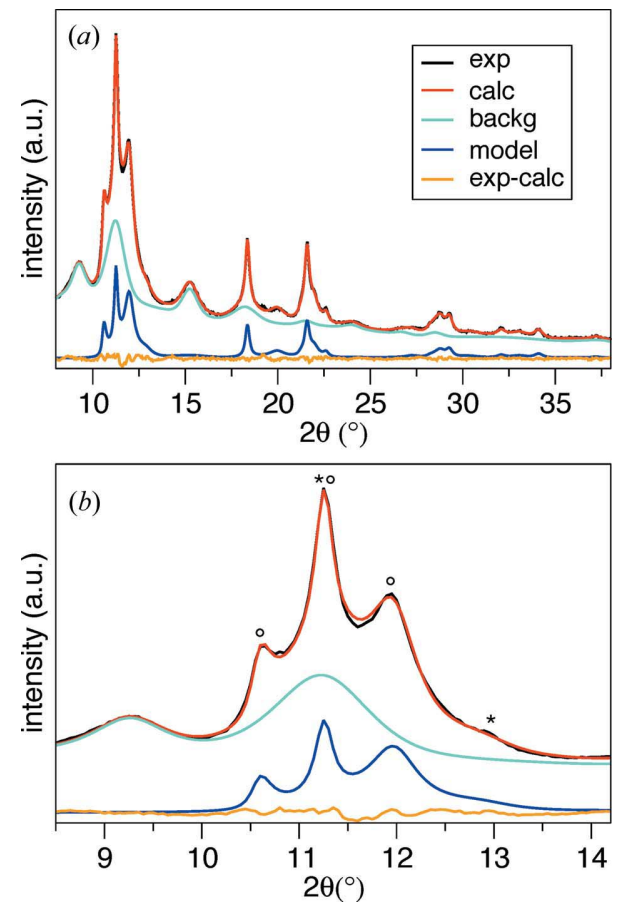

Figure 3

H sample. (a) Fitting of the model $s=4$ in the $8.5-38^{\circ} 2 \theta$ range $(\lambda=0.4 \AA$ monochromatic X-ray radiation). (b) Enlargement relative to the $2 \theta$ region most sensitive to the close-packing arrangement. Open circles: h.c.p. signatures; stars: c.c.p. signatures. The model contribution is rescaled by 0.5 .
It is worth noting that the model does not take into account the possibility, indeed quite reasonable, of a dependence of the disorder parameters on particle size, so that the optimized values of $\alpha, \beta, \gamma$ and $\delta$ should be considered as averages taken over the statistical ensemble of lognormal-distributed crystallites; moreover, it is possible that very small clusters adopt noncrystalline structures (Cervellino et al., 2003). Also, these limitations can be easily overcome, but, as previously observed, this involves going beyond the actual amount of information inherent in the experimental data.

\subsection{Simulations of $X$-ray absorption data}

The atomic coordinates for the theoretical EXAFS and XANES simulations were generated as follows: close-packed layers of Co atoms were stacked according to the four different four-layer blocks (cc, ch, hc and hh) weighted by the respective frequencies and concatenated with $\mathrm{c}$ or $\mathrm{h}$ according
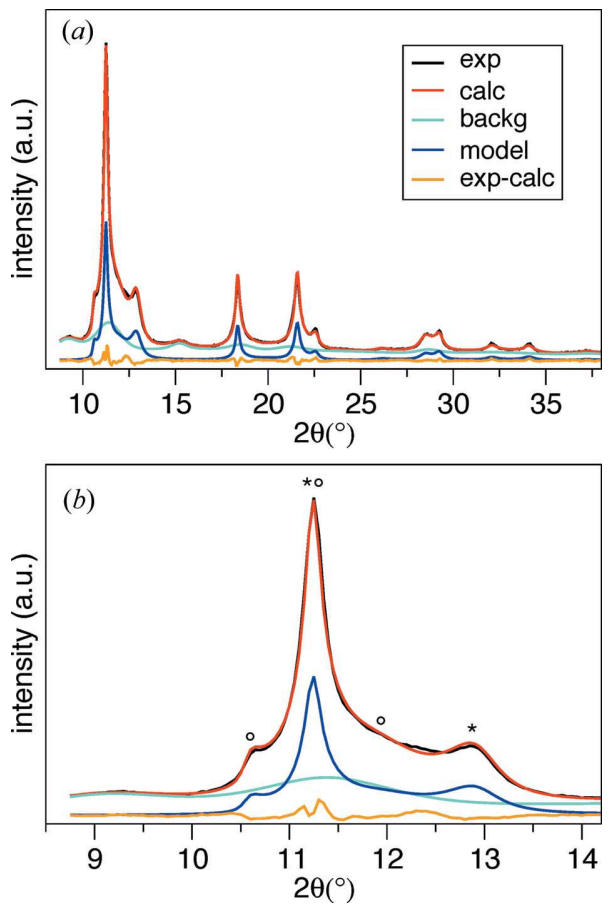

Figure 4

C sample. (a) Fitting of the model $s=4$ in the $8.5-38^{\circ} 2 \theta$ range $(\lambda=0.4 \AA$ monochromatic X-ray radiation). (b) Enlargement relative to the $2 \theta$ region most sensitive to the close-packing arrangement. Open circles: h.c.p. signatures; stars: c.c.p. signatures. The model contribution is rescaled by 0.5 . 


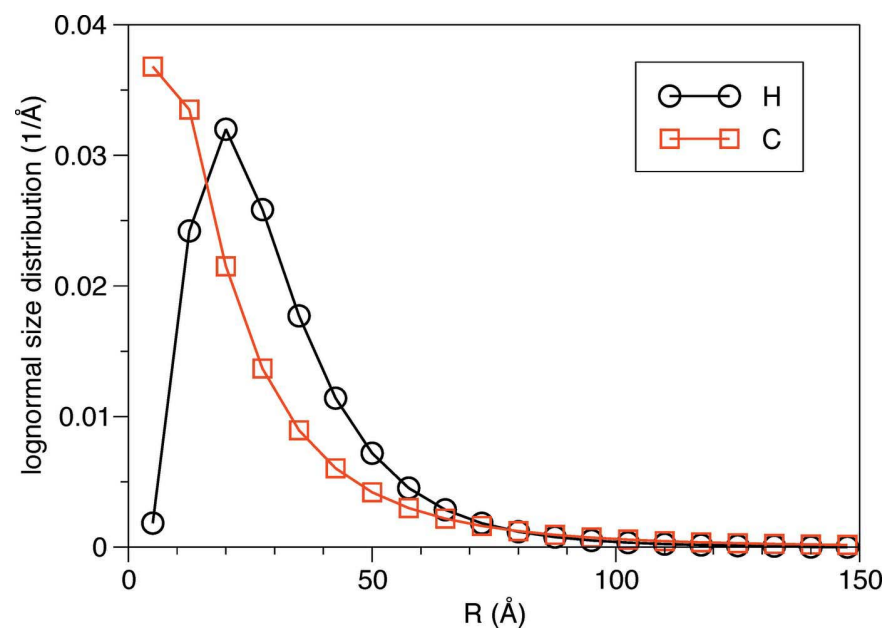

Figure 5

Lognormal size distribution for $\mathrm{H}$ and $\mathrm{C}$ samples. The respective average radius and standard deviation are reported in Table 2.

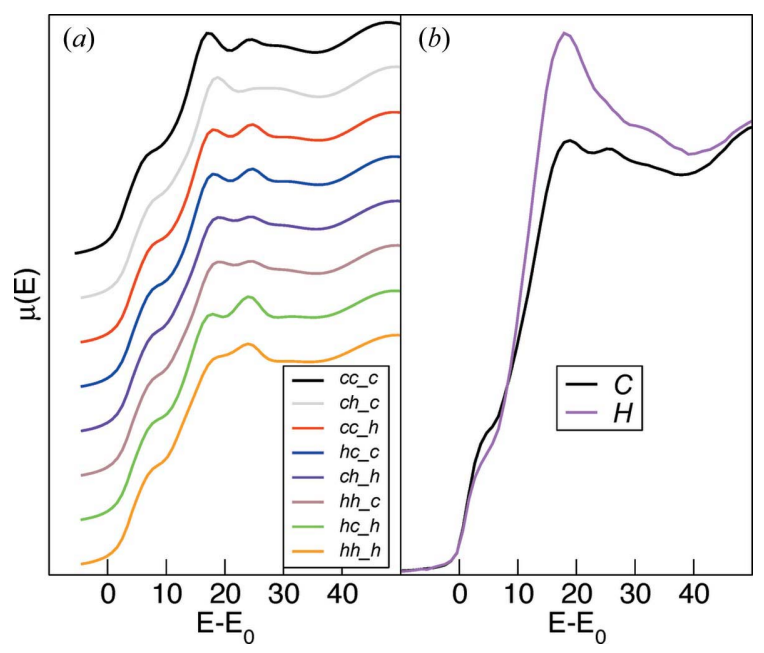

Figure 6

(a) Simulated XANES spectra of the five-layer sequences specified in the legend; the spectra are multiplied by 0.5 and shifted. (b) Experimental data of the $\mathrm{C}$ and $\mathrm{H}$ samples.

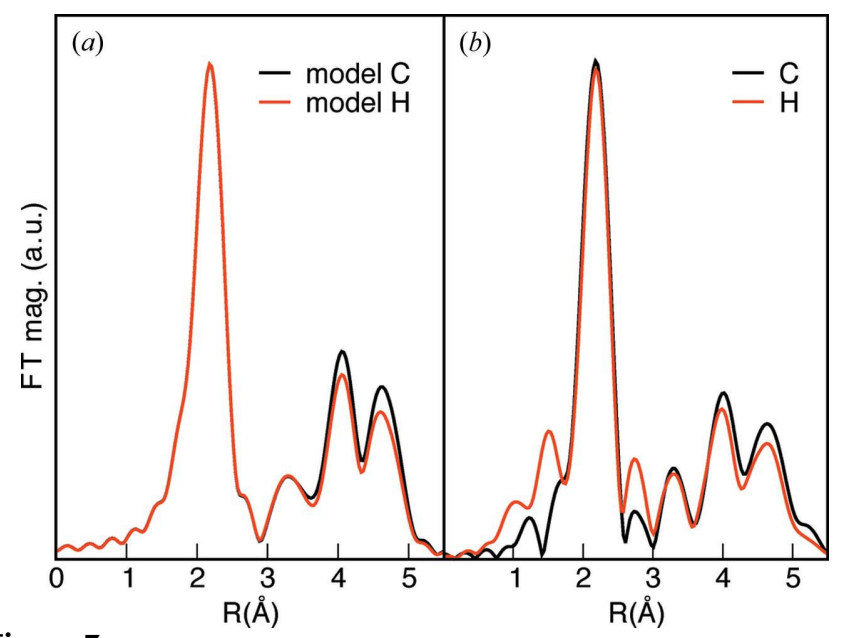

Figure 7

(a) FT magnitude of EXAFS spectra simulated with the parameters reported in Table 2 for the $\mathrm{H}$ and $\mathrm{C}$ samples. (b) Experimental data. to the sequence probabilities reported in Table 2. So, eight different five-layer slabs are built. For instance, the statistical weight of the hc_c slab is given by $f_{\mathrm{hc}} \beta$, that of hc_h is $f_{\mathrm{hc}}(1-$ $\beta$ ) and so on. Then, the sum over the eight different slabs equals one. The absorber atom was placed at the centre of each slab, and the spectra corresponding to each slab were calculated with Feff (Ankudinov et al., 1998). For XANES, the spectra were calculated with a full multiple scattering (FMS) radius of $6 \AA$ around each atom and the Hedin-Lundqvist exchange potential with $1 \mathrm{eV}$ experimental broadening. For EXAFS, the calculation allowed for static and vibrational disorder by using a correlated Debye model plus a constant Debye-Waller factor of $0.003 \AA^{2}$. The simulations corresponding to samples $\mathrm{H}$ and $\mathrm{C}$ were obtained by a weighted average of the EXAFS signals of the component sequences, using the parameters refined through the XRD fitting (Table 2), an FMS radius of $6 \AA$ and a $1 \mathrm{eV}$ Hedin-Lundqvist exchange potential.

Fig. 6(a) reports the XANES spectra simulated from the five-layer slabs described above, while in Fig. 6(b) the experimental spectra of the $\mathrm{H}$ and $\mathrm{C}$ samples are drawn.

It is worth noting that the fine details of the simulated spectra can be recognized in the experimental XANES data reported in the literature (Sprouster et al., 2009); the comparison with the experimental $\mathrm{C}$ sample shows a clear f.c.c.-like habitus, probably modified by the minor oxide component also recognized in the corresponding XRD data. On the other hand, the $\mathrm{H}$ sample data are strongly affected by the oxide component, whose presence is characterized by a prominent white line. The EXAFS Fourier transform (FT) magnitudes are reported in Fig. 7(b), while Fig. 7(a) shows the averages of the five-layer slabs obtained using the four-layer frequencies and continuation probabilities reported in Table 2.

The $\mathrm{H}$ sample data show the presence of the $\mathrm{Co}-\mathrm{O}$ shell on the low- $R$ side of the main Co-Co first-neighbour metal shell. The other features of the EXAFS FT of both samples do not show clear evidence of further shells from the oxide phase, confirming its amorphous structure. It is worth noting that the difference between the calculated $\mathrm{H}$ and $\mathrm{C}$ patterns can be recognized in the comparison between the experimental data, in particular as concerns the third and fourth shells in the 3.5$5 \AA R$ interval.

\section{Conclusions}

This paper analyses two snapshots of nanosized cobalt in the boundary region where c.c.p. and h.c.p. sequences coexist. A structural model characterized by a statistical correlation range of four atomic layers accounts for the main features of the respective XRD patterns. It is likely that most of the literature diffraction data relative to nanosized cobalt showing the concurrent presence of h.c.p. and c.c.p. signatures can be fitted in the framework of this model. The assumption that a range of correlation is effective introduces in a completely random close packing a degree of local order, so endorsing the theoretical analysis that the f.c.c. and h.c.p. phases of cobalt evolve, with different growth mechanisms, from a disordered 
parent polytype constituted by the alternation of ordered blocks and disordered stacking regions (Tolédano et al., 2001). The fitting to XRD data provides an estimate of the relative abundance of building blocks, which can be correlated with experimental results from local structural probes like NMR and EXAFS, and with $a b$ initio computational analyses of magnetic and catalytic properties.

The technical assistance of the staff of beamlines BM26 and ID31 of ESRF is gratefully acknowledged.

\section{References}

Ankudinov, A. L., Ravel, B., Rehr, J. J. \& Conradson, S. D. (1998). Phys. Rev. B, 58, 7565-7576.

Barcaro, G., Sementa, L., Negreiros, F. R., Ferrando, R. \& Fortunelli, A. (2011). Nano Lett. 11, 5542-5547.

Cervellino, A., Giannini, C. \& Guagliardi, A. (2003). J. Appl. Cryst. 36, 1148-1158.

Chakroune, N., Viau, G., Ricolleau, C., Fiévet-Vincent, F. \& Fiévet, F. (2003). J. Mater. Chem. 13, 312-318.

Debye, P. (1915). Ann. Phys. 46, 809-823.

Diehl, M. R., Yu, J., Heath, J. R., Held, G. A., Doyle, H., Sun, S. \& Murray, C. B. (2001). J. Phys. Chem. B, 105, 7913-7919.

Ducreux, O., Rebours, B., Lynch, J., Roy-Auberger, M. \& Bazin, D. (2009). Oil Gas Sci. Technol. Rev. IFP, 64, 49-62.

Ferrando, R., Rossi, G., Nita, F., Barcaro, G. \& Fortunelli, A. (2008). ACS Nano, 2, 1849-1856.

Gnanamani, M. K., Jacobs, G., Shafer, W. D. \& Davis, B. H. (2013). Catal. Today, 215, 13-17.

Hakamada, M., Hirashima, F., Kajikawa, K. \& Mabuchi, M. (2012). Appl. Phys. A, 106, 237-244.

Jagodzinski, H. (1949). Acta Cryst. 2, 208-214.

Jun, Y. W., Seo, J. W. \& Cheon, J. (2008). Acc. Chem. Res. 41, 179-189.

Kakinoki, J. (1967). Acta Cryst. 23, 875-885.

Leslie-Pelecky, D. L., Bonder, M., Martin, T., Kirkpatrick, E. M., Liu, Y., Zhang, X. Q., Kim, S. \& Rieke, R. D. (1998). Chem. Mater. 10, 3732-3736.

Li, Y., Liu, Q. \& Shen, W. (2011). Dalton Trans. 40, 5811-5826.

Lisiecki, I. \& Pileni, M.-P. (2011). J. Phys. Chem. C, 116, 3-14.

Liu, Z., Chang, P., Chang, C., Galaktionov, E., Bergmann, G. \& Lu, J. G. (2008). Adv. Funct. Mater. 18, 1573-1578.

Liu, J. X., Su, H. Y., Sun, D. P., Zhang, B. Y. \& Li, W. X. (2013). J. Am. Chem. Soc. 135, 16284-16287.

Longo, A. \& Martorana, A. (2008). J. Appl. Cryst. 41, 446-455.
Lu, A., Salabas, E. \& Schüth, F. (2007). Angew. Chem. Int. Ed. 46, 1222-1244.

Margeat, O., Amiens, C., Chaudret, B., Lecante, P. \& Benfield, R. E. (2005). Chem. Mater. 17, 107-111.

McHenry, M., Majetich, S., Artman, J., DeGraef, M. \& Staley, S. (1994). Phys. Rev. B, 49, 11358-11363.

Niederdraenk, F., Seufert, K., Luczak, P., Kulkarni, S. K., Chory, C., Neder, R. B. \& Kumpf, C. (2007). Phys. Status Solidi C, 4, 3234 3243.

Pankhurst, Q. A., Thanh, N. T. K., Jones, S. K. \& Dobson, J. (2009). J. Phys. D Appl. Phys. 42, 224001.

Peña O'Shea, V. A. de la, de la Piscina, P. R., Homs, N., Aromí, G. \& Fierro, J. L. G. (2009). Chem. Mater. 21, 5637-5643.

Popa, N. C. \& Balzar, D. (2002). J. Appl. Cryst. 35, 338-346.

Puntes, V. F., Krishnan, K. M. \& Alivisatos, A. P. (2001). Science, 291, 2115-2117.

Puntes, V. F., Zanchet, D., Erdonmez, C. K. \& Alivisatos, A. P. (2002). J. Am. Chem. Soc. 124, 12874-12880.

Qiao, R., Zhang, X. L., Qiu, R., Li, Y. \& Kang, Y. S. (2007). J. Phys. Chem. C, 111, 2426-2429.

Rempel, A. \& Magerl, A. (2010). Acta Cryst. A66, 479-483.

Schio, P., Bonilla, F. J., Zheng, Y., Demaille, D., Milano, J., de Oliveira, A. J. \& Vidal, F. (2013). J. Phys. Condens. Matter, 25, 056002.

Sciortino, L., Giannici, F., Martorana, A., Ruggirello, A. M., Turco Liveri, V., Portale, G., Casaletto, M. P. \& Longo, A. (2011). J. Phys. Chem. C, 115, 6360-6366.

Sokalski, V., Laughlin, D. E. \& Zhu, J. (2011). J. Appl. Phys. 110, 093919.

Sort, J., Nogués, J., Suriñach, S., Muñoz, J. \& Baró, M. (2004). Mater. Sci. Eng. A, 375-377, 869-873.

Sort, J., Suriñach, S., Muñoz, J., Baró, M., Wojcik, M., Jedryka, E., Nadolski, S., Sheludko, N. \& Nogués, J. (2003). Phys. Rev. B, 68, 014421.

Speight, R., Wong, A., Ellis, P., Bishop, P. T., Hyde, T. I., Bastow, T. J. \& Smith, M. E. (2009). Phys. Rev. B, 79, 054102.

Sprouster, D., Giulian, R., Schnohr, C., Araujo, L., Kluth, P., Byrne, A., Foran, G., Johannessen, B. \& Ridgway, M. (2009). Phys. Rev. B, 80, 115438.

Srivastava, A. K., Madhavi, S. \& Ramanujan, R. V. (2010). Appl. Phys. A, 98, 821-830.

Tolédano, P., Krexner, G., Prem, M., Weber, H. \& Dmitriev, V. (2001). Phys. Rev. B, 64, 144104.

Xu, R., Xie, T., Zhao, Y. \& Li, Y. (2007). Cryst. Growth Des. 7, 1904 1911.

Xu, Y., Mahmood, M., Li, Z., Dervishi, E., Trigwell, S., Zharov, V. P., Ali, N., Saini, V., Biris, A. R., Lupu, D., Boldor, D. \& Biris, A. S. (2008). Nanotechnology, 19, 435102.

Zhao, L. J., Duan, L. F., Wang, Y. Q. \& Jiang, Q. (2010). J. Phys. Chem. C, 114, 10691-10696. 